\title{
Gene Expression Is Disrupted by Ethanol Exposure on Chick Embryonic Choroid Plexus
}

\author{
Xing Kang1, Weerapong Prasongchean ${ }^{1,2 *}$ \\ ${ }^{1}$ Department of Biochemistry and Microbiology, Faculty of Pharmaceutical Sciences, Chulalongkorn \\ University, Thailand. \\ ${ }^{2}$ Chulalongkorn University Drug and Health Products Innovation and Promotion Center, Chulalongkorn \\ University, Thailand.
}

* Corresponding author. Tel.: +662-218-8371; email: weerapong.p@chula.ac.th

Manuscript submitted November 12, 2018; accepted January 23, 2019.

doi: 10.17706/ijbbb.2019.9.3.141-149

\begin{abstract}
Alcohol is a well-known teratogenic agent that causes neurodevelopmental diseases, including fetal alcohol syndrome. Alcohol-induced brain changes such as neuronal cell death, decreased adult neurogenesis, low level of neurotrophic factors and vital nutrients were previously reported. The choroid plexus (CP) is a multifunctional tissue located in four ventricles of the brain and formed early during neural development. The CP play important roles in producing cerebrospinal fluid, supplying numerous neurotrophic factors and eliminating brain metabolites, supporting homeostasis of central nervous system (CNS) both pre and postnatally. Involvement of the CP in alcohol-induced brain damage is yet to be discovered. This study aimed to investigate effects of alcohol on the developing CP of the lateral ventricles of the chick embryonic brains. We started treatments of alcohol $(10 \% \mathrm{v} / \mathrm{v}$ and $15 \% \mathrm{v} / \mathrm{v})$ in ovo chick embryos at embryonic day 3 (E3) every 24 hours and the CP examined at E8 and E12. Ethanol exposure induced growth retardation of the CP at E8 and E12 (decrease in both length and size). We then found that the mRNA expression of Otx2, Emx1 and Emx2 were up-regulated by ethanol exposure, and E2f5 mRNA was reduced and Foxj1 mRNA was increased expression in the CP of alcohol-treated embryos was confirmed. Herein, the current study showed the first time that alcohol disrupt the CP development related genes might lead to abnormal and dysfunction of the developing CP during early brain development, suggesting a pharmaceutical target for prevention and treatments of neurodevelopmental diseases.
\end{abstract}

Key words: Choroid plexus, fetal alcohol syndrome, gene expression.

\section{Introduction}

Generally, alcohol has been reported as embryonic teratogen that may lead to many congenital malformation diseases in various organs, which is commonly named as fetal alcohol syndrome (FAS). Especially, constellations of developmental disorders were characterized by growth and mental retardation in pre- and post-natal organs, such as brain malformation [1], central nervous system (CNS) anomalies [2], and craniofacial malformations [3]. Overall, these structural deficits can occur at different stages of embryonic development that also underlie the functional deficits occur on brain causing neurodevelopment disorder diseases. However, the diverse cellular and molecular mechanisms of FAS by which ethanol exert its developmental toxicity, as teratogen is unclear yet. Moreover, some by-products also can be generated from ethanol metabolism, which also is other aspect to express the teratogenic property of alcohol, such as reactive oxygen species (ROS), acetaldehyde, lipid peroxidation products and protein adducts [4]. 
The choroid plexus (CP) is a multifunctional tissue that resides in all these four cerebral ventricles, which is responsible for both producing cerebrospinal fluid (CSF), containing numerous neurotrophic factors, and eliminating brain metabolites and waste for central neural system (CNS) homeostasis and neurogenesis in brain development [5]. Apparently, the CP is tree-like structure wrapping with a single layer of cuboidal epithelium (CP epithelium, CPE), and is continued into the ependyma lining the two laterals, third and fourth ventricles of brain. In addition, the inner CP stromal core is generated from the mesenchyme as an origin, which consist of a core of fenestrated capillaries from the endothelium. In chick embryos, the $\mathrm{CP}$ is formed at around embryonic day 6 (E6) - 8 (E8). We showed previously the presence of neurons, neural progenitors within the $\mathrm{CP}$, and nerve innervation can occurs prenatally [6]. Importantly, CP generates molecular cues that guide regulatory components expression and local interactions during embryonic neural development [7]. Previous studies have demonstrated that choroid plexus also has been reported to closely relate within these neural diseases [8], and the choroid plexus epithelium paly important role for secreting glutathione contained CSF to protect the nervous system health [9].

We ask whether brain damage from alcohol exposure can be indirectly resulted from effects of ethanol on the developing CP. Therefore, the present work aims to examine alcohol effects on CP morphological changes during embryonic development, explore a possible gene expression that might be beneficial for the prevention or treatments of neurodevelopmental diseases.

\section{Materials and Methods}

\subsection{Animals}

All fertilized white leghon eggs were obtained from the Department of Animal Science, Faculty of Agriculture, Kasetsart University, Bangkok, Thailand. In brief, eggs measured the weight approximately 45 to 50 grams on balance, and cleaned with $70 \%$ ethanol were transferred and maintained in the incubator at $38.5^{\circ} \mathrm{C}$ with $60 \%$ humidity until reaching required embryonic stages. All procedures were carried out under Thailand Experimental Animals for Scientific Purposes Act.

\subsection{Methods}

\section{- Treatment of Chemicals}

Embryo viability was confirmed by windowing eggs. Then eggs were randomly assigned to different groups ( $n>20$ per group), control (DI water), $10 \%$ v/v EtOH, $15 \%$ v/v EtOH. Injection of $200 \mu$ l of solutions was done on the air space of eggs at embryonic day 3 (E3) and repeated everyday until E8 and E12 [10]. Survival of embryos was monitored.

\section{- Isolation and histological study of the Brain and the Choroid Plexus (CP)}

Live embryos at required stages were collected and gross anatomy examined. Brains were isolated, and weight and size measured. The $\mathrm{CP}$ of the brain lateral ventricle was isolated from forebrain for further experiment. In addition, the whole brain was fixed in 4\% paraformaldehyde (PFA) in phosphate-buffered saline (PBS) overnight at $4^{\circ} \mathrm{C}$ and washed with PBS 3 times for histological study. In brief, brains were dehydrated in $30 \%$ sucrose solution, embedded in optimal condition temperature (OCT) solution (Sakura

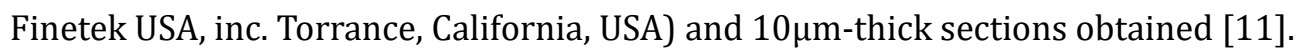

\section{- Hematoxylin \& eosin staining}

A serial OCT-embedded brain sections was sectioned using Microtome and stained with hematoxylin and eosin (Bio-Optica Milano SpA, San Faustino, Italy). Briefly, the sections were removed OCT by rinsing xylene. The sections were hydrated by passing through decreasing concentration of alcohol baths and water $(100 \%$, $90 \%, 80 \%$, and $70 \%$ ). The sections were stained with hematoxylin for 3-5 minutes, and washed in running tap water until sections "blue" for 5 minutes or less. The sections were stained in $1 \%$ Eosin $Y$ for 10 minutes 
and washed in tap water for 1-5 minutes. Finally, sections were dehydrated by increasing concentration of alcohols and clear in xylene, mounted in mounting media and observed under stereomicroscope.

\section{- Reverse transcription - polymerase chain reaction (RT-PCR)}

In order to detect the special transcription factors presence in CP tissue and assess whether the different expression by ethanol treatment. Total RNA samples would be extracted from lateral ventricle CP in E8 and E12 using TRIzol reagent according to the protocol of the manufacturer (Life Technologies, 5791 Van Allen Way, Carlsbad, CA, USA). A $2 \mu \mathrm{g}$ amount of total RNA was transcribed reversely into cDNA by using KAPA2G Fast HotStart ReadyMix Kit (Tiangen, Beijing, China) in a total volume of $25 \mu \mathrm{l}$. The primers were designed for chick species and summarize as the Table 1, and were amplified using these primers with 35 cycles. The PCR products were fractionated on agarose gel and visualized by ethidium bromide staining. Semi-quantitative gene expression data were normalized using GAPDH housekeeping gene as an internal control to elucidate the ratio of relative band intensity of target gene. The band intensity of ethidium bromide fluorescence was measured using an ImageJ analysis system, then quantified with SPSS 21.0 statistical software.

Table 1. List of Primers Used in Semi-quantitative RT-PCR of Transcription Factors mRNAs of Embryonic Choroid Plexus

\begin{tabular}{|c|c|c|c|c|}
\hline Gene & Sequences & $\begin{array}{l}\text { Product } \\
\text { (bp) }\end{array}$ & $\begin{array}{l}\text { Internal standard } \\
\text { /Target }\end{array}$ & $\begin{array}{l}\text { Genebank } \\
\text { reference }\end{array}$ \\
\hline Otx2 & $\begin{array}{l}\text { Forward CCACCTCAACCAGTCTCCAG } \\
\text { Reversed TTCCATGAGGATGTCTGGTC }\end{array}$ & 168 & Target & NM_204520.2 \\
\hline Emx1 & $\begin{array}{l}\text { Forward GGCGGACGAAATACAAACGG } \\
\text { Reversed CCTGCCTTAGTCGTTGGAGG }\end{array}$ & 152 & Target & XM_001232150.5 \\
\hline Emx2 & $\begin{array}{l}\text { Forward GCAGCGGAAGGACACGTAGA } \\
\text { Reversed CGGTAGCGGTGTATTAGCCA }\end{array}$ & 571 & Target & XM_015867460.1 \\
\hline E2f5 & $\begin{array}{l}\text { Forward ATGACCTTGCACAAACCCCA } \\
\text { Reversed AGACACCTACCAAGAGTGCTG }\end{array}$ & 467 & Target & NM_001030942.1 \\
\hline Foxj1 & $\begin{array}{l}\text { Forward GCAGAACTCCATCCGACACA } \\
\text { Reversed GGTTTGGTTCGGTCCTTTGC }\end{array}$ & 828 & Target & NM_001321535.1 \\
\hline GAPDH & $\begin{array}{l}\text { Forward CCAGGTTGTCTCCTGTGACT } \\
\text { Reversed CACAACACGGTTGCTGTAT }\end{array}$ & 202 & Internal & NM_204305.1 \\
\hline
\end{tabular}

\section{- Statistical analysis}

The experimental values were recorded as means with standard deviation, statistical difference between control and other experimental groups were evaluated with Turkey and LSD test of post-hoc multiple comparisons of one-way ANOVA using SPSS 21.0 software, and setting statistical significance at ${ }^{*} \mathrm{p}<0.05$.

\section{Results}

\subsection{Ethanol Induces Brain Growth Retardation}

To investigate the correlation between ethanol treated and natural embryonic brain development, $10 \%$ and $15 \%(\mathrm{v} / \mathrm{v})$ ethanol were administrated to chicken embryos starting from E3 to E8, and morphological evaluation of E8 brain was presented in Fig. 1 A-C and E12 brain was presented in Fig. 1 A'-C'. The isolated embryonic brain of each group had a normal anatomic morphology, including forebrain (FB), midbrain (MB), cerebellum (CB), and spinal cord [2]. However, both doses ethanol treated E8 embryonic brains represented growth retardation in the forebrain region with smaller size when compared with control group, and more apparent size reduction was observed from 15\% ethanol group. In addition, the weights of embryonic brain were collected to interpret the quantitative analysis of growth restriction. Both ethanol treated embryos were found few weight decreasing when compared with control group at E8 stage, and 
embryonic brain weight were observed the 5\% decreasing on $10 \%$ ethanol treated group and $11 \%$ decreasing in 15\% ethanol treated group (Fig. 1 D), Nonetheless, the undramatic results were found as growth retardation on embryonic brain on E8 stage. Differently, the both ethanol treated E12 brain represented smaller size of forebrain region, and brain tissue were sloppy comparing with control. Especially, the hemorrahge of forebrain and midbrain region also be observed in $15 \%$ ethanol treatment group. Moreover, both of 10\% and 15\% ethanol treated of E12 brain weights were significantly decreased when compared with the control group, which were shown as the $15 \%$ weight reducing in $10 \%$ ethanol treated group and the $21 \%$ weight reducing in $15 \%$ ethanol treated group (Fig. $1 \mathrm{E}$ ).
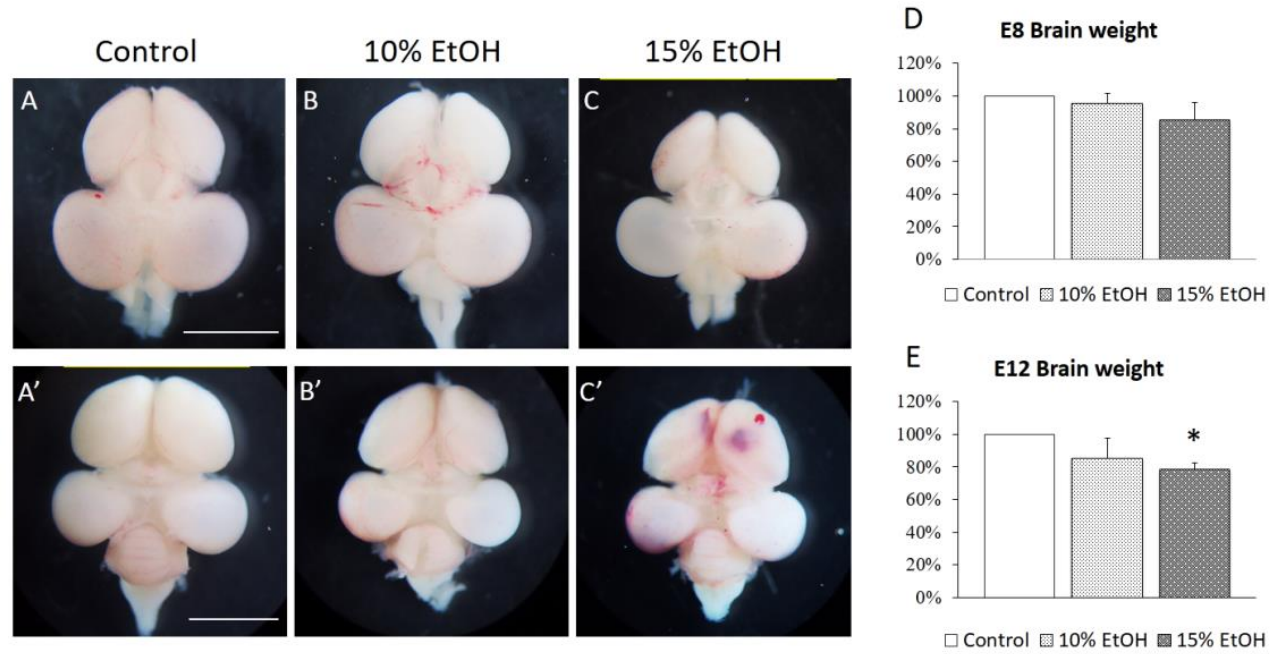

Fig. 1. Morphological effects of ethanol on CP at embryonic day 8.

E8 embryonic brain morphology changes with different treatment were presented under optical microscope (A-C). E12 embryonic brain morphology changes with different treatment were presented under optical microscope $\left(A^{\prime}-C^{\prime}\right)$. Brain size decreasing were presented against $10 \%$ and $15 \%$ ethanol treatment at both E8 and E12 stages. The percent change of brain weight were significantly reduced by $15 \%$ ethanol treatments comparing to control at E12 stage (D and E) $\left({ }^{*} p<0.05\right)$.

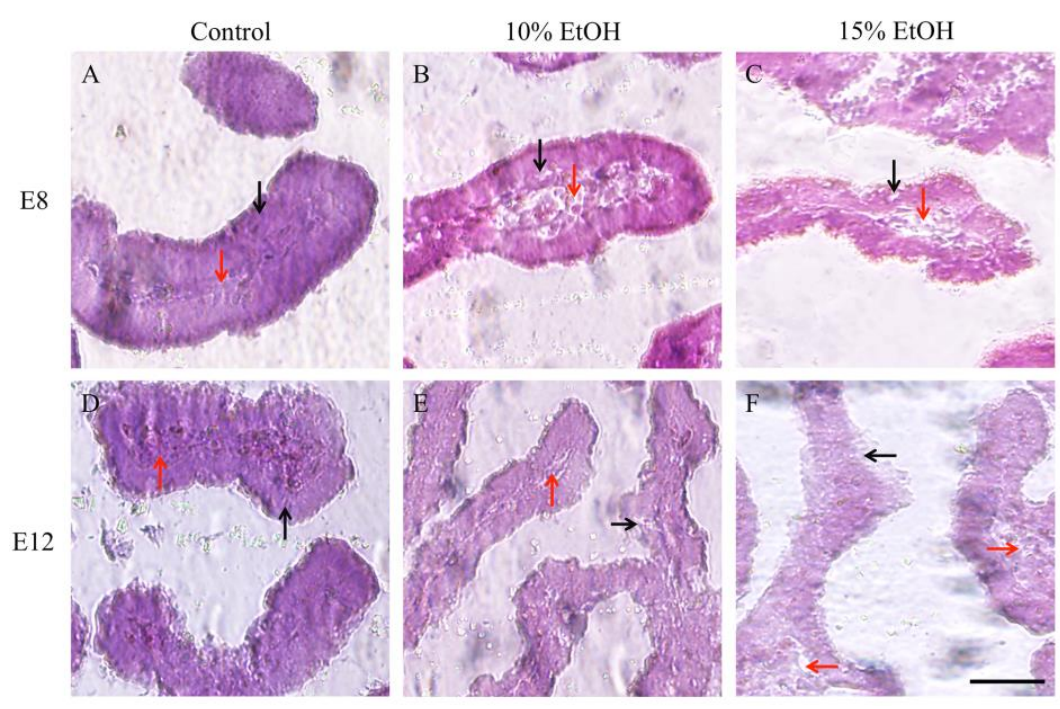

Fig. 2. H\&E staining of E8 and E12 chick embryonic CP. 
The histological analysis of E8 and E12 CP, 10\% ethanol-treated (B and E) and 15\% ethanol-treated (C and F) experimental groups with abnormal structure of CP comparing control (A and D), black arrows point out the abnormality in epithelium layer, and red arrows refer to the disruption on stomal and blood vessel regions. scale bar $=50 \mu \mathrm{m}$.

\subsection{Ethanol Induces Histological Change on Choroid Plexus}

The histological effects of ethanol exposure on development of choroid plexus using H \& E staining were shown in Fig. 2, these observations were more considerable in CP structure and epithelium layer of ethanol-treated groups compared to control group.In the control groups, E8 CP were represent a more moisture and freshly trunk, also stromal and blood vessels were normal and tight junction each other, while abnormal distribution of $\mathrm{CP}$ epithelium layer were found more gap without staining, the damaged connective tissue and incompact blood vessels were observed in experimental groups (Fig. 2 B and C). Moreover, the similar abnormalities also were observed in E12 ethanol-treated CP comparing to control. Interestingly, the $\mathrm{CP}$ epithelium layer also represented a reduced thickness in somewhere, which was more considerable for the fact of abnormal CP induced by ethanol exposure (Fig. $2 \mathrm{E}$ and F).

\subsection{Ethanol Disrupted Gene Expression on Choroid Plexus}

To find a suitable genetic marker that reflects the normal developmental pattern of choroid plexus during embryogenesis, we have analyzed the expression pattern of the selected transcription factor mRNAs (otx2, emx1, emx2, e2f5, foxj1) during CP of chick embryogenesis by applying semi- quantitative reverse transcription - polymerase chain reaction (RT-PCR) using total RNA extracted from the whole amount of chick lateral ventricle choroid plexus at E8 and E12 stages, which the gel electrophoresis figures of mRNA expression were recorded (Fig. $3 \mathrm{~A}$ ).
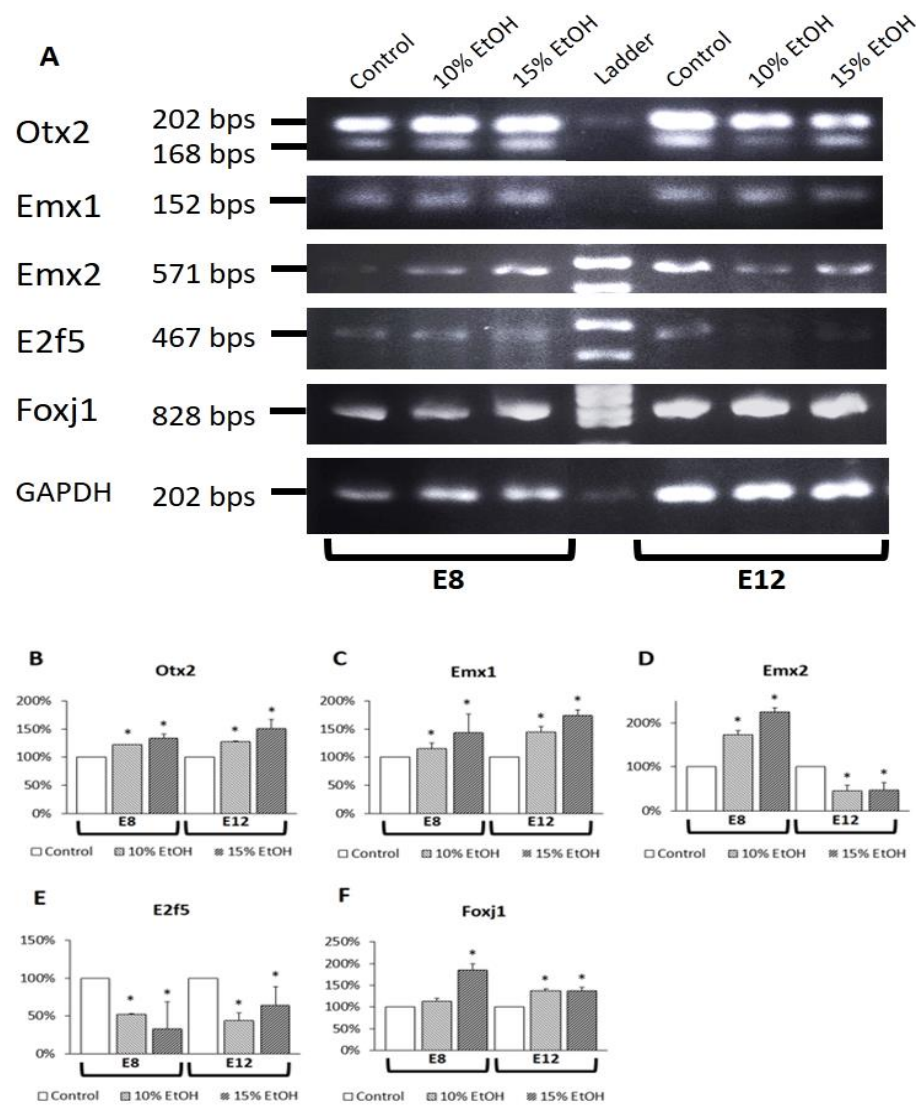

Fig. 3. Gene expression alteration on the alcohol exposed chick embryonic CP. 
The reverse transcription PCR of Otx2, Emx1, Emx2, E2f5, Foxj1 and GAPDH on chicken embryos represented on gel figure (A). The E8 gene expression were on left panel of ladder and E12 gene expression were on right panel of ladder. Percentage changes of each gene expression were compared with control group to represent as the ratio of relative band intensity of target gene: GAPDH (B-F). The ratio data obtained were transformed and used for one way ANOVA followed by post-hoc Turkey's multiple comparison test, which a significant difference among groups were marked $*(P<0.05)$.

Each mRNA of control embryonic CPs considered as E8 and E12 stages was prepared from the embryos treated with sterilized DI water only. In semi-quantitative RT-PCR analysis, the mRNA level of all these transcription factors were represented in all kinds of treatment of embryonic CP at both E8 and E12 stages which indicate these mRNA were involved CP development (Fig. 3 B - F). Interestingly, the mRNA E2f5 was observed the band region surrounding the target band that expressed as early as cleavage started on both E8 and E12 stages (Fig. 3 E). In case of ethanol treated embryonic CP, the mRNA expression of Otx2, Emx1, Foxj1 were up-regulated by ethanol on both E8 and E12 (Fig. 3 B, C and F), but the Emx2 was down-regulated especially on E12 (Fig. 3 D). The opposite or indifferent mRNA expression of E2f5 was observed comparing with control groups on E8 and E12 stages (Fig. 3 E). However, even ethanol was unable to show same effects of disruption in the mRNA expression pattern of all these target genes, but the similar mRNA expression of GAPDH were observed on ethanol and any substances treated CP, that represented a stabile expression to be internal standard gene for investigating the ratio results of each target mRNA expression and relative interactions.

\section{Discussion}

\subsection{Alcohol-Induced Embryonic Choroid Plexus Abnormality Underlying Neurodevelopmental Diseases}

Chicken embryos were chosen as an optimal model system for these kinds of study as advantages of less time consuming, easy handling, without maternal sacrifice, and reliable results as Clemens's summarization [3]. We started alcohol treatment at E3 that cause the central nervous system (CNS) is being developed at this stage. The alcohol-induced embryonic brain malformation was observed by using chicken embryos via morphological and physical analysis in this study. The main finding was observed that weight of both does ethanol treated E12 chicken brains were significantly reduced comparing with control, to be consistent for any teratogenesis occurring with ethanol exposure (Fig. 1). However, there was not any significant evidence of weight reducing to be found on E8 ethanol treated brain. This reliable result was not only consistent for previous studies performed on human and chick embryo [1], which showed analogical brain abnormalities of last trimester of gestation underlying fetal alcohol syndrome (FAS), but it also estimates the brain is particularly sensitive to ethanol causing long-term effects [12].

As the aim of this study, abnormal CP is caused by prenatal ethanol exposure that is a main risk factor for neurodevelopmental diseases as central nervous system (CNS) damages [13]. Our results showed that histology of choroid plexus were disrupted on both E8 and E12 stages (Fig. 2). The morphology and function of choroid plexus may be regulated in response to various physiological or pathophysiological parameters during embryonic development [14]. However, there is few evidence that introduce quantitative development of $\mathrm{CP}$ [14] and elucidate the features of fetal CP ultrastructural changes in fetal alcohol syndrome [15], and results were similar to adults CP of alcoholism, and were summarized as severe deformations of CPE mitochondria, enlargement of the intercellular space and separation of the basolateral interdigitations between the cells of the CP epithelium [16]. Interestingly, one original research also have demonstrated the alcohol induced dysfunction adult CP can lead to significant pathology of CSF secretion and alteration of neurotrophic contains elsewhere in the brain, which suggests the alcohol induced 
dysfunction CP can be a major factor to cause other neurological diseases also [17]. All these above evidences of abnormalities of embryonic CP demonstrated that underdeveloped embryonic brain is caused by alcohol-induced growth retardation at early stages of development, which early $\mathrm{CP}$ would be vulnerable to alcohol for a possible and initial event in neurodevelopmental diseases.

\subsection{Alteration of Gene Expression Is a Reason for Abnormal Choroid Plexus Development.}

This study has shown that the transcripts for Otx2, Emx1/2, E2f5, Foxj1 are expressed through out the developing lateral ventricle $\mathrm{CP}$ in chick embryos, indicating that their expression is controlled at the transcriptional level. The mRNA expression of Otx2, Emx1 and Emx2 were up-regulated that represented on $10 \%$ and 15\% ethanol treated CP comparing with control. Upon deletion of Otx2 by induction of recombination at E9 mouse and complete absence of Otx2 stained positive cell by E11, all CP failed to form [18]. However, another CP surrounding structure (neuroepithelial tissue) and cortical hem still expresses Otx2 without effect. Thus, Otx2 is not vital for the hem only, but it is also required for CP development [19]. These data revealed Otx2 as a master regulator of CP development, it being absolutely essential for these tree-like structures to appear. Strikingly, this role remains when Otx2 mRNA expression is reduced before E14 or E15 lateral ventricle CP and relative CP length were subsequently decreased [20]. Indeed, our results represented an increasing Emx1 and Emx2 mRNA expression on ethanol treated embryonic CP at E8 and E12 stages (Fig. $3 \mathrm{C}$ and D). Emx genes play a more general role to promote cell division that reduced proliferation and lengthening of the cell cycle in the Emx2 negative cortex [21]. While Emx1/2 miss-expression leads to down-regulation of Otx2, BMP7 and Ttr inhibits the acquisition of cell biological $\mathrm{CP}$ characteristics, such as the differentiation into a thin epithelial tissue [19]. However Otx2 was already expressed in early highly proliferating tissue, at very early stages even together with Emx2 [22]. In contrast, Otx2 up-regulation were observed to overexpress Emx1 and/or Emx2 mRNA in lateral ventricles CP is rather linked to alteration of cell fate than the higher proliferation rate. Thus, this would contribute a possible mechanism that the relative expression levels of Emx genes adjust the euroepitheliual tissue for positioning the CP. In index, the high Emx1 and/or Emx2 expression levels were represented in the midline region (hem) to shift Otx2 expression then via lowing endogenous Emx expression levels in CP [19]. E2f5 and Foxj1 transcription factors have been reported a lacking functional in mouse model that subsequently induced non-obstructive hydrocephalus [23], which cause the mutated foxj1 expression to induce the absence of ciliated epithelia of body presenting in CP, and the E2f5 knockout mice were observed CP epithelium containing more electro-lucent cells to indicate a high secretory activity than normal [24]. Although our results showed that E2f5 mRNA was reduced and Foxj1 mRNA was increased expression by ethanol treatment (Fig. $3 \mathrm{E}$ and F). In index, a decreasing of E2f5 at late stage of gestation has been reported in the rat cortex and PC12 cells to induced for undergoing neural differentiation [25], and high level of E2f5 mRNA in early developing mouse nervous system (E9.5) when the neuronal differentiation was activated [26]. Especially, aberrance higher expression Foxj1 also has been reported to represent on low-grade ependymal and choroid plexus tumors, which abnormal cilia might provide signals analogous to those that result during the disassembly and re-sorption of cilia [27].

\section{Conclusion}

The CP plays important roles in brain development and homeostasis. Changes in CP structures and functions would result in the altered brain physiology, might lead to teratogenicity or neurodevelopmental disorders. In this study, we tested whether alcohol affect the development of the CP in the chicken embryonic model. Altogether, alcohol elevated the important transcription factors have been investigated to elucidate a relative malformed and dysfunctional CP occurrence via various genes expression. 
Pharmacological intervention could reverse these toxic effects. Insights into functional alterations of the CP and the brain are essential for further investigation, suggesting new therapeutic target for FAS.

\section{Acknowledgment}

This research is supported by the 90th Anniversary Chulalongkorn University Rachadapisek Sompote Fund, Chulalongkorn University. We also thank Pharmaceutical Research Instrument Center, Faculty of Pharmaceutical Sciences, Chulalongkorn University and Chulalongkorn University Drug and Health Products Innovation and Promotion Center.

\section{References}

[1] (2015). Liddelow SA: Development of the choroid plexus and blood-CSF barrier. Front Neurosci, 9, 32.

[2] Danielyan, L., et al. (2009). Intranasal delivery of cells to the brain. Eur J Cell Biol, 88(6), 315-324.

[3] Carvan, M. J., et al. (2004). Ethanol effects on the developing zebrafish: Neurobehavior and skeletal morphogenesis. Neurotoxicology and teratology, 26(6), 757-768.

[4] Brocardo, P. S., et al. ( 2011). The role of oxidative stress in fetal alcohol spectrum disorders. Brain Res Rev, 67(1-2), 209-225.

[5] Tan, R. R., et al. (2013). Glucose metabolism disorder is a risk factor in ethanol exposure induced malformation in embryonic brain. Food and Chemical Toxicology: An International Journal Published for the British Industrial Biological Research Association, 60, 238-245.

[6] Prasongchean, W, et al. (2015). The neural milieu of the developing choroid plexus: neural stem cells, neurons and innervation. Frontiers in Neuroscience, 9, 103.

[7] Redzic, Z. B., et al. (2005). The choroid plexus-cerebrospinal fluid system: from development to aging. Current Topics in Developmental Biology, 71, 1-52.

[8] Kaur, C., Rathnasamy, G., \& Ling, E. A. (2016). The choroid plexus in healthy and diseased brain. J Neuropathol Exp Neurol, 75(3), 198-213.

[9] Aliaghaei, A., Khodagholi. F., \& Ahmadiani, A. (2014). Conditioned media of choroid plexus epithelial cells induces Nrf2-activated phase II antioxidant response proteins and suppresses oxidative stress-induced apoptosis in PC12 cells. J Mol Neurosci, 53(4), 617-625.

[10] Smith, S. M., Flentke, G. R., \& Garic, A. (2012). Avian models in teratology and developmental toxicology. Methods in Molecular Biology, 889, 85-103.

[11] Jaszai, J., et al. (2013). Spatial distribution of prominin-1 (CD133)-positive cells within germinative zones of the vertebrate brain. PLoS One, 8(5), e63457.

[12] Miller, M. W. (2007). Exposure to ethanol during gastrulation alters somatosensory-motor cortices and the underlying white matter in the macaque. Cereb Cortex, 17(12), 2961-2971.

[13] Heaton, M. B., et al. (2000). Ethanol-induced alterations in the expression of neurotrophic factors in the developing rat central nervous system. Brain Res Dev Brain Res, 121(1), 97-107.

[14] Stastny, F., \& Rychter, Z. (1976). Quantitative development of choroid plexuses in chick embryo cerebral ventricles. Acta neurologica Scandinavica, 53(4), 251-259.

[15] Craciun, V., et al. (1989). Ultrastructural changes in the mouse fetal choroid plexuses following chronic maternal alcoholization.

[16] Tirapelli, L. F., Tamega, O. J., \& Petroni, S. (2000). Ultrastructural alterations of choroid plexuses of lateral ventricles of rats (Rattus norvegicus) submitted to experimental chronic alcoholism. Arquivos de Neuro-Psiquiatria, 58(1), 25-31.

[17] Nixon, P. F., et al. (2008). Choroid plexus dysfunction: the initial event in the pathogenesis of Wernicke's encephalopathy and ethanol intoxication. Alcohol Clin Exp Res, 32(8), 1513-1523. 
[18] Barkho, B. Z., \& Monuki, E. S. (2015). Proliferation of cultured mouse choroid plexus epithelial cells. PloS One, 10(3), e0121738.

[19] Frowein, J., Wizenmann, A., \& Gotz, M. (2006). The transcription factors Emx1 and Emx2 suppress choroid plexus development and promote neuroepithelial cell fate. Developmental Biology, 296(1), 239-252.

[20] Johansson, P. A., Irmler, M., \& Acampora, D., et al. (2013). The transcription factor Otx2 regulates choroid plexus development and function. Development, 140(5), 1055-1066.

[21] Muzio, L., \& Mallamaci, A. (2003). Emx1, emx2 and pax6 in specification, regionalization and arealization of the cerebral cortex. Cereb Cortex, 13(6), 641-647.

[22] Bell, E., et al. (2001). Dynamic domains of gene expression in the early avian forebrain. Developmental Biology, 236(1), 76-88.

[23] Yang, A., et al. (2000). p73-deficient mice have neurological, pheromonal and inflammatory defects but lack spontaneous tumours. Nature, 404(6773), 99-103.

[24] Lindeman, G. J., et al. (1998). A specific, nonproliferative role for E2F-5 in choroid plexus function revealed by gene targeting. Genes Dev, 12(8), 1092-1098.

[25] Persengiev, S. P., et al. (1999). E2F4 actively promotes the initiation and maintenance of nerve growth factor-induced cell differentiation. Mol Cell Biol, 19(9), 6048-6056.

[26] Dagnino, L., et al. (1997). Expression patterns of the E2F family of transcription factors during mouse nervous system development. Mechanisms of Development, 66(1-2), 13-25.

[27] Chen, H. W., et al. (2013). Expression of FOXJ1 in hepatocellular carcinoma: correlation with patients' prognosis and tumor cell proliferation. Mol Carcinog, 52(8), 647-659.

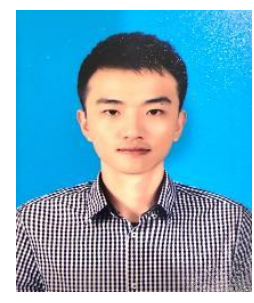

Xing Kang was born on June 4, 1989 in Kunming, Yunnan, China. Recently, Mr. Xing is a master degree student at Faculty of Pharmaceutical Sciences, Chulalongkorn University from Chulalongkorn University, who is professional and focusing at Neuroscience, Developmental biology, Toxicology, etc. Previously, Mr. Xing graduated with a bachelor degree in Biotechnology concerning the plant molecular genetics research at Mae Fah Luang University in Thailand.

He has completed his internship as a assistant researcher since march/2013 to May/2013 under supervision of Professor Dr. Amorntip Muangprom in the Plant Molecular Genetics Laboratory, Agricultural Biotechnology Research Unit of BIOTEC, National Center for Genetic Engineering and Biotechnology (BIOTEC), Thailand. The field of studies are about that many available SSR markers was investigated the possibility to identify hybridized rice among hundreds cultivars.

Importantly, Mr. Kang also has attend many professional conferences passionately to share his onw research for various audiences. Mr. Kang has been selected for inclusion in the program as a poster presentation, which it was evaluated and scored by the Abstract Review Panel in the 12th International Conference on Cerebral Vascular Biology (CVB2017), Melbourne, AUS. 